\title{
LABORATORY FUNDAMENTAL DATA
}

\author{
W. R. S. GARTON \\ Physics Dept., Imperial College of Science and Technology, London, England
}

An essential prescription for a review paper prepared for a conference is that it treat an area of currently flourishing research - a requirement directly conflicting with limitations of time and space. Under the present title, a large output of diverse and enterprising experimental endeavour has indeed appeared in the three years since the Maryland meeting, so that the reviewer is forced to be very selective. Fortunately, the situation is alleviated by the inclusion in Dr Burgess's review of 'Plasma Spectroscopy' of much material describable as 'fundamental data' - such as $f$-value measurements obtained from plasma sources, shock-tubes and arcs. Again little reference is needed here to the important and extensive work on line identifications and classifications in spectra of medium to high ionization stages, since these will be covered by $\mathrm{Dr} C$. Jordan's review and several contributed papers.

With the benefit of such relief the present review is planned to concern:

(1) Brief note of recent advances in XUV technology and some notable experiments based thereon, with note of early expectations of technical and instrumental advance.

(2) Selected topics on atomic and molecular spectra, autoionization effects and absorption cross-sections, drawn from work of the last few years.

(3) Some aspects of solid-state UV spectroscopy of likely astrophysical relevance.

(4) The little explored effects of atomic diamagnetism, recently observed spectroscopically in the ultraviolet.

\section{Technology, etc.}

The rapid progress which began in the 1950's has continued, and notable advances in instrumental capabilities and in auxiliary apparatus and techniques have been reported - especially as regards light-sources. As a result, a number of significant researches, to be mentioned later, have been either greatly facilitated or made possible for the first time.

The central item for the laboratory XUV specialist remains the concave grating, and most workers in this region continue in debt to the Bausch \& Lomb Company for its contributions over twenty years. These benefits will be illustrated a little later by reference to the performance of one outstanding diffraction grating from this source.

The important matter of the provision of gratings of exceptional performance, size, or both, together with the advance of the ruling art by traditional means - has depended for years on the activities of the laboratory of G. R. Harrison at MIT (Harrison and Thompson, 1970). Reassuring information has been received in recent months that this important field of development work will continue from 1971 or 1972 at Kitt Peak National Observatory, following Dean Harrison's retirement. 
As regards the equally important - indeed, for some participants in the present conference more significant - matter of grazing-incidence rulings, some very recent developments are to be noted. For some years, Franks (1970) of the National Physical Laboratory in England has been advancing the qualities of grazing-incidence rulings through the application of ingenious etching techniques, by which he has produced so-called 'phase gratings'. These have proved to have good efficiencies at extreme grazing angles and correspondingly short wavelengths. However, till just recently, though individual gratings thus produced were known to be very good, lack of time and/or facilities hindered systematic investigation of the effects of surface finish and groove-profile on luminous efficiencies. Since the Maryland meeting, Speer (1970) of Imperial College has installed, - in collaboration with UKAEA and ARU Culham and the National Physical Laboratory, - a large double monochromator of great versatility for grating evaluation. A diagram of the layout of this instrument is shown in Figure 1. In Figure 2 are shown the results of some measurements, versus spectral order, of efficiencies of one particular grating at two angles. Notable features are the peculiar disposition of this grating, (presumably not unique), to favour the first negative order, and the small angular range over which this disposition occurs. It may be noted also that the energy in the first negative order for this grating exceeds by a factor greater than two that in any other order. These results represent the first part of what is expected to be an extended programme of grating evaluation, (Speer, 1971).

A possibility of an entirely new dispersive technique for very short wavelengths is currently being explored by Dr J. Bahr of Imperial College (unpublished). The basic idea is that for photons of high energy in the XUV - say corresponding to less than $20 \AA$,-- resolving powers of both gratings at grazing-incidence and crystal spectrometers are very low as compared to what is acceptable in the normal-incidence range above $200 \AA$. On the other hand, low-energy beta-ray spectroscopy, as practised for instance in electron-'eigenlos' experiments, is generally more impressive - for example,

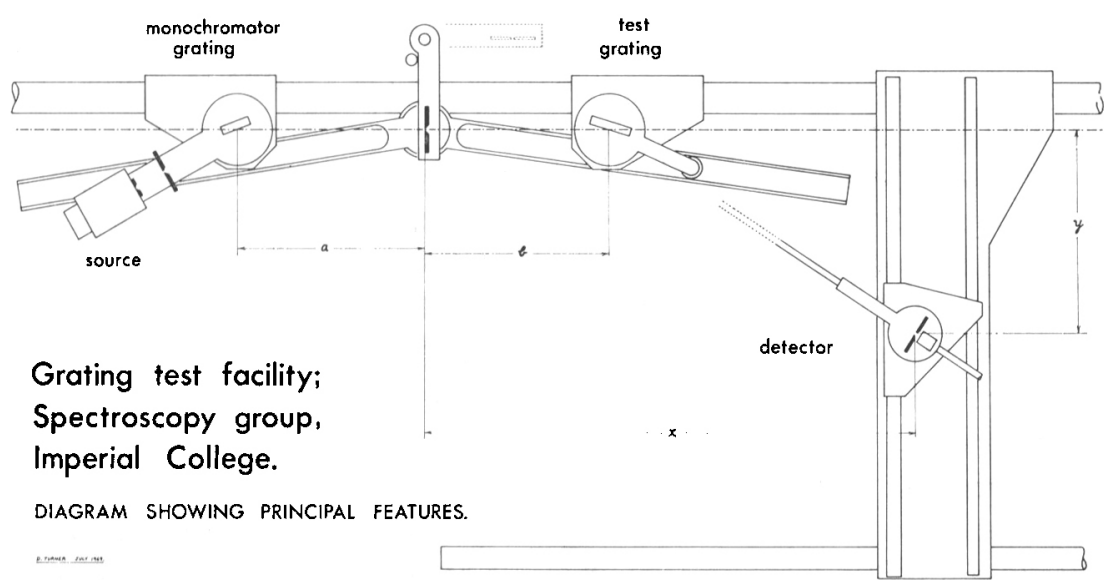

Fig. 1. Principal features are shown. Grating test facility; Spectroscopy group, Imperial College. 

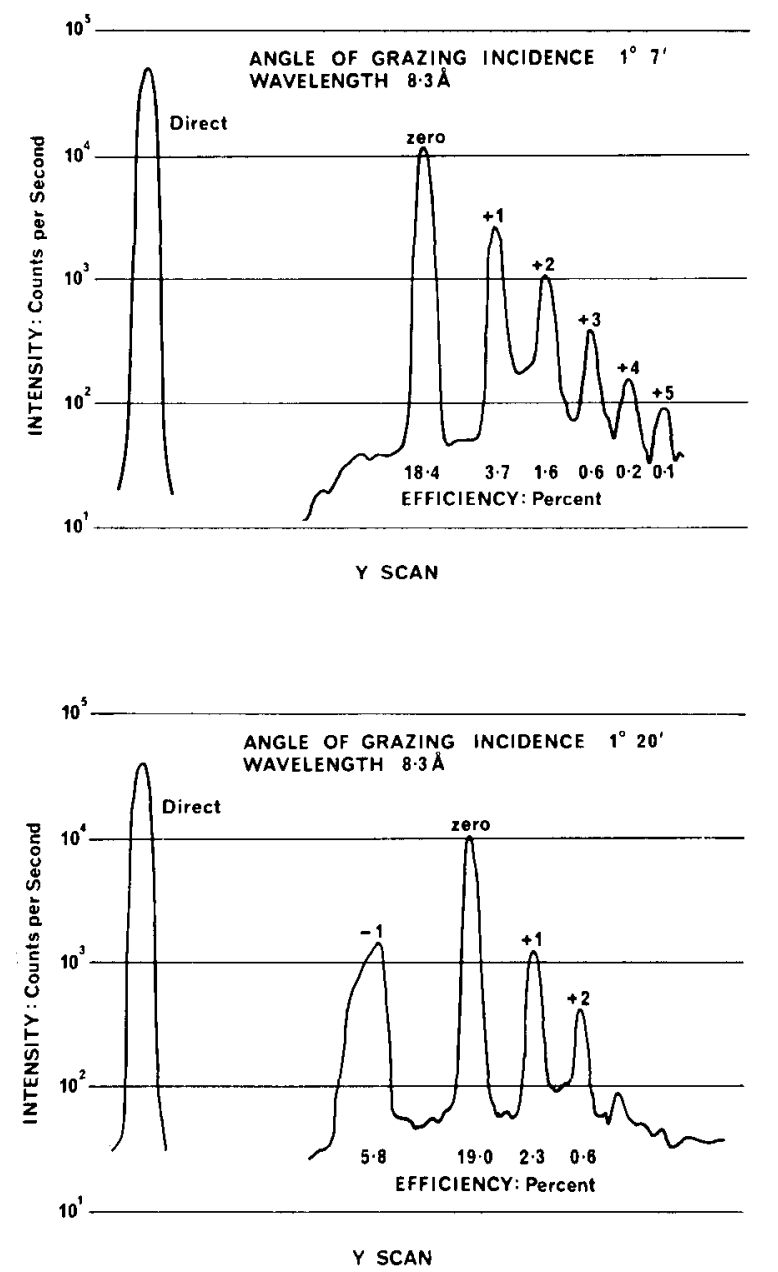

Fig. 2. Distribution of luminous efficiency vs spectral order for a grazing-incidence grating.

electron beams of some tens of kilovolt energy can penetrate solids or gases, lose energies measured in tens of $\mathrm{eV}$, and be energy-analyzed to better than $10 \mathrm{mV}$. Dr Bahr's experiments are aimed at ascertaining whether the energy-analysis of photoelectrically-ejected electrons from a target gas can be used to determine the spectrum of the incident radiation; that is, he is attempting to use photoelectron spectroscopy 'in reverse', so to speak. It is as yet too early to know whether this possibility of new dispersive instrumentation, applicable at very short wavelengths, will be successful.

On the other hand, great promise attaches, for the ultraviolet generally, - and especially for the short wavelength end and soft $X$ rays, - to the development of the 'holographic grating'; work in this direction has been flourishing recently at the JobinYvon Company in France (Labeyrie and Flamand, 1969; Cordelle et al., 1969; also 
Beesley and Casteldine, 1970). The basic idea of recording, by photo-chemical means, an interferogram of controllable pattern on an optical surface, - plane or concave, was initiated quite a long time ago: for instance, Burch and Palmer (1961) were doing exploratory experiments ten to fifteen years ago. However, proper development of the possibility had to await the emergence of laser technology. Diffraction gratings produced in this way promise advantages in size, beam aperture and narrow ruling interval, together with freedom from stray light and ghosts. Moreover, they offer the possibility of aberration-corrected spectra over useful wavelength ranges, - for example in grazing-incidence use the possibility of zero astigmatism.

As regards light sources, Dr Burgess has dealt with a number of successful applications of several plasma devices, including shock-tubes, and stabilized arcs, of importance for the generation and identification of line spectra of various stages of ionization, for the determination of $f$ values and of collision cross-sections, and in the study of line-broadening mechanisms. It is here only necessary to supplement his report with reference, for the most part, to light sources which are not classified as 'plasmas'. A justifiably exception to this limitation is, however, found in an experimental tour de force by Marrone and Wurster (1971). These workers employed a fast-acting (explosively-driven) valve in front of a spectrometer with entrance-slit mounted in the wall of a pressure-driven shock tube; they were able to use the assembly in the windowless region below the LiF cut-off and to a wavelength as short as $700 \AA$. The photoelectric spectrometer was used for measurements of spectral intensity distributions in the emission continua generated in reflected shocks in $\left(\mathrm{Ne}+\mathrm{N}_{2}\right)$ and $(\mathrm{Ne}+\mathrm{CO})$, at plasma temperatures of about $13000^{\circ}$. These measurements thus yielded recombination cross-sections for the processes $\mathrm{N}^{+}+e \rightarrow \mathrm{N}+h v$, etc., and conversely, photoionization cross-sections of importance to the physics of stellar atmospheres, interstellar space and planetary atmospheres.

The 'beam-foil' technique has been very vigorously developed during the past three years, as illustrated at the 1970 conference in Stockholm reported in Nucl. Instr. Methods 90, December 1970. A major school of activity continues at Tucson under $\mathrm{S}$. Bashkin, who remarked at the 1970 meeting that over twenty laboratories were then engaged and it was difficult to keep track of papers even in this specialized field. The same group contributed significantly to the problem at the Spring 1971 Optical Society of America Meeting by participating in seven out of nine contributions! There has, indeed, been a large output of new work reported in this field during the past twelve months. The initial ambition of the beam-foil experimenters, up to about 1967-68, seems to have been directed towards extensive determinations of lifetimes of energy levels of highly-ionized species, which were in demand for both laboratory and observational astrophysics. Up to much the same date, nearly all the work was confined to the range of the visible spectrum but, by 1970, we find the whole range from the infrared to soft X-ray region opened up to this sort of experiment. Thus, Bashkin (1970) estimates that over forty elements have been variously studied over this wide spectral range, with incident particle energies ranging from $10 \mathrm{kV}$ to $400 \mathrm{MeV}$. In parallel with the extension of spectral range, the objectives of work in this field have 
widened to include, not only lifetime measurements, but the location and wavelength measurement of previously unknown transitions, - e.g. in the light elements. Also, ingenious experiments on beams in externally applied fields have been reported (e.g. Liu et al., 1971). A few citations from recent papers or conference contributions illustrate these points and the current vigour of beam-foil spectroscopy.

With regard to measurement of lifetimes of energy levels yielding transitions in the XUV, Martinson (1971) reports measurements in light species, He I, II, O III, IV in the range $200-600 \AA$; the radiation was excited by $2 \mathrm{MeV}$ beams of $\mathrm{He}^{+}$and $\mathrm{O}^{+}$. This worker has identified interesting transitions from non-autoionizing doubly-excited levels like $2 p 3 p^{1} P_{1}, 2 p^{2}{ }^{3} P$; measured lifetimes are in good agreement with calculations made recently by Drake and Dalgarno (1970). Again, lifetime measurements for Si II, III, IV levels made by Bromander et al., (1971) at Stockholm extend to $800 \AA$ and include Si II, $3 p^{2} P^{0}-3 d^{2} D$ at $1261 \AA$ which is supposed strongly present in quasar spectra. The Tucson group has recently worked down to a few Ångtrom, and initial experiments were announced this year, (Bickel et al., 1971; Garcia, 1971). These experiments have been, in part, aimed at studies of the mechanism of the production of $\mathrm{X}$ radiation at, and close to the foil, during the passage of the beam, with a view to visualizing the manner of excitation of inner-shell electrons of the beam-ions. In this connection we note that Dufay (1970) remarks on the possible importance of autoionization, in beam-foil excitation processes, on the basis of the occurrence of transitions between doubly-excited energy-levels of Li I (Buchet et al., 1969; Bickel et al., 1969).

Turning now to sources of continua needed for investigations of the absorption spectra of gases, and in work on the detail of absorption and reflection spectra of solids, we note that, during the period since the Maryland meeting, the Bureau of Standards work under Madden and Ederer has continued, following re-installation of the $180 \mathrm{MeV}$ electron-synchrotron. This apparatus has been used to carry forward the celebrated work of the earlier 1960's by Madden, Codling and Ederer on the absorption spectra of the inert gases (Madden and Codling, 1966). The same laboratory has also been engaged in measurements of the optical properties of solids under ultra-high vacuum conditions. At least three other synchrotrons are in current use as light sources at this date, - namely at Hamburg, Bonn and in Japan, - with some work still in progress with the useful machine at Glasgow. Note should also be made of the intention to use the high-energy Daresbury accelerator in the near future. To date, most of the publications, reporting employment of synchrotron radiation, - aside from the work on the inert gases (Madden and Codling, 1966), have reported results on the optical properties of solids. An ambitious enterprise (Gahwiller et al., 1970), which has now come to fruition after several years of hope and endeavour, has been started at Madison Wisconsin, in the exploitation of a $240 \mathrm{MeV}$ storage ring as a light-source. This apparatus is currently probably the most stable and intense source of continuum for the range $40-400 \AA$. The synchrotron light is so intense that it can be used for simultaneous experiments on radiation damage and XUV spectroscopy. Experiments recently reported again mainly concern solid-state work. 
On much less ambitious scale the 'synchrotron monopoly', which certainly prevailed in absorption spectroscopy of gases at wavelengths below $600 \AA$ a few years ago, has been at least reduced by the development of the 'BRV source'. This is very much a 'table-top' device, which we owe to the ingenuity of the group at the Bellevue Laboratory of Professor Vodar (Balloffet et al., 1961; Lotte et al., 1963; Damany et al., 1966). The 'BRV source' appears to be essentially a miniature 'Filippov-pinch' or 'plasma-focus' (Mather, 1964), and yields a very usable continuum, probably emitted by the focussed plasma at the tip of the Uranium rod employed as anode. At Imperial College, J. E. G. Wheaton has, we think, improved some details of design and operation, and that laboratory has been using the 'BRV source' very successfully for observation of a number of important atomic and molecular absorption spectra to be referred to later. In Figure 3 the effect of varying the anode material (Wheaton, un-

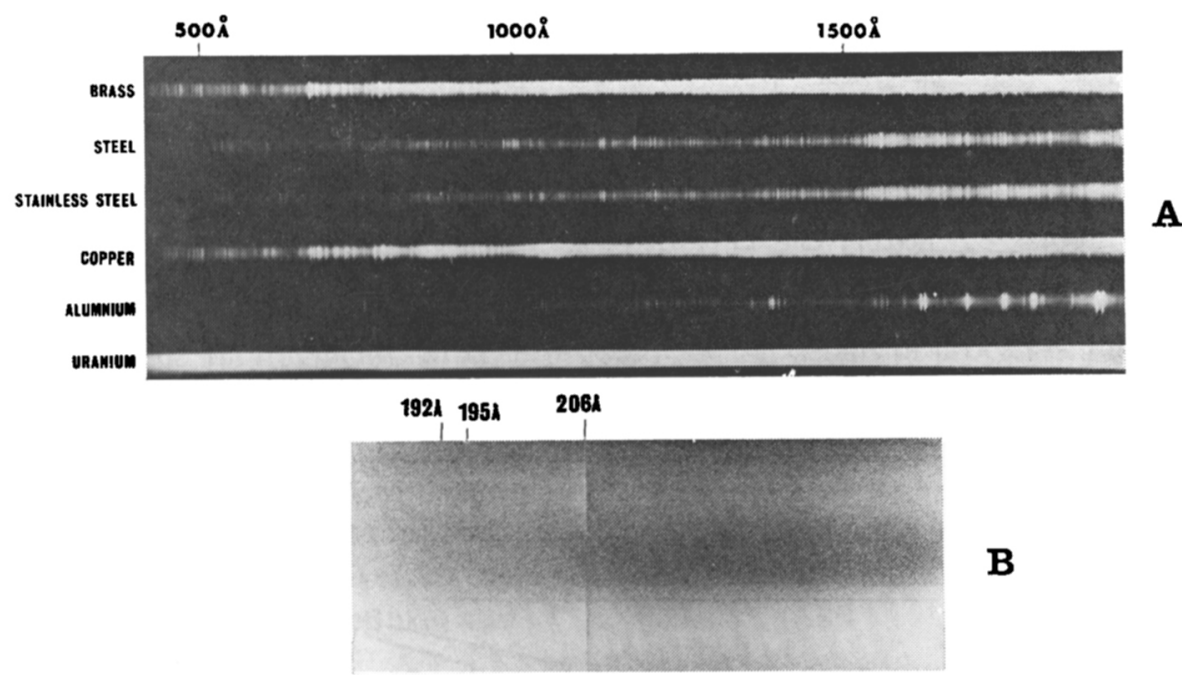

Fig. 3. Spectra obtained with 'BRV-source': (A) Spectra of source with various anodes - (B) The He $1 s^{2}{ }^{1} S_{0} \rightarrow 2 s 2 p^{1} P_{1}^{0}$ and other resonances in absorption near $200 \AA$ (3-metre normal-incidence).

published), and the use of this light source for recording several of the He absorption resonances in the neighbourhood of $200 \AA$ (cf. Madden and Codling, 1965), are shown.

The final matter of technique to be discussed here concerns the generation and containment of atomic and molecular species in absorption work in the windowless region below $500 \AA$, where it is no longer feasible to use helium as a buffer gas. This problem was reported already solved in principle at the Maryland meeting, by the use of low pressure He and systems of cooled baffles (Garton et al., 1969). In very recent NBS work on $\mathrm{Li}$ and Na Madden's group have used thin windows, - eg: of $\mathrm{Al}$ and $\mathrm{C}$,- on a grazing-incidence mounting, combined with as short a path, and as 
much He pressure as tolerable, together with a 'heat-pipe', (Grover et al., 1964; Eastman, 1968). Some of the results are referred to below.

In connection with absorption spectroscopy at short wavelengths, it is worth noting a recently revived, - and seemingly valuable, - technique known as 'flash-pyrolysis'. This technique was introduced by Nelson and co-workers about ten years ago (Nelson and Kuebler, 1962; Kuebler and Nelson, 1961), chiefly for studies of metallurgical interest. The method resembles the more familiar combination of flash-absorption spectroscopy and 'flash photolysis', save that the reaction vessel contains initially a finely-divided solid material instead of a gas. In some experiments about ten years ago (and unpublished), by Dr F. S. Tomkins and the author at Argonne National Labo-

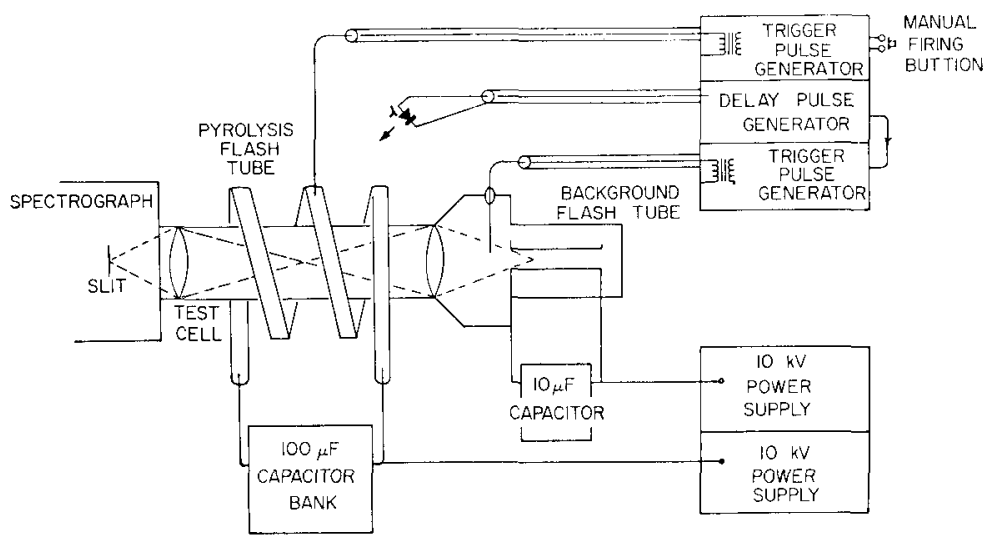

Fig. 4. Block diagram of 'Flash-pyrolysis' apparatus.

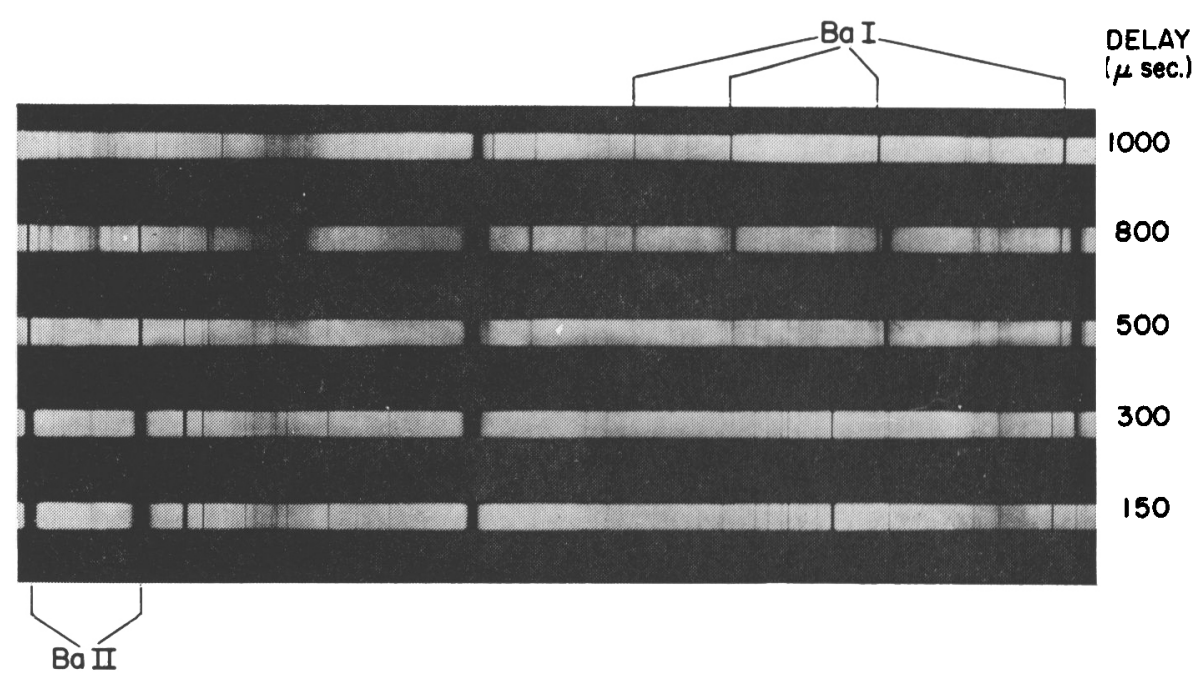

Fig. 5. Effect of delay time on barium absorption. 
ratory, the method was used in an attempt to obtain the absorption spectra of $\mathrm{Ra} I$. Though no particular success attached to this effort, an interesting observation was made, during trial runs with barium, - namely that, during the first hundred microseconds or so following the 'pyrolyzing flash', not only was finely-divided $\mathrm{BaCl}_{2}$ fully dissociated, but the resulting $\mathrm{Ba}$ atoms were almost fully ionized. Figure 4 shows a block diagram of the 'flash-pyrolysis' apparatus. Dr Tondello describes, in these conference proceedings, the successful application of this technique for the production of the important S I absorption spectrum, and the Harvard group (Parkinson, Reeves and Tondello, unpublished) are using the same system in attempts to observe the absorption spectra of other neutrals of higher ionization potential and of ions of low ionization potential. Figure 5 which shows the absorption spectrum of barium, obtained at Argonne in the early work, (i.e. about eight years ago), illustrates the change of degree of ionization vs time.

\section{Atomic and Molecular Absorption Spectra}

Under this heading a good deal has been accomplished, and space permits mention of only a small selection of the new work. Thus, there is space to notice only in passing the programme of elegant work by Chupka and Berkowitz and their colleagues at Argonne, on photoionization cross-sections of some atoms and of a wide range of molecular species, combined with photoelectron spectroscopy and mass-spectrometric analysis of the ion fragments. Many cross-sections and details of processes, including autoionization structures, have been described in a large number of papers, chiefly in the Journal of Chemical Physics over the last few years.

A notable recent success of the revived programme of work with the NBS synchrotron has been observation of the Li I absorption spectrum in the XUV (Ederer et al., 1970). This simple atom gives a by no means simple spectrum in the range below $250 \AA$, due to excitation processes like:

$$
1 s^{2} 2 s^{2} S_{1 / 2} \rightarrow 1 s 2 s n l \text { and } 1 s n s n^{\prime} l
$$

WAVELENGTH $(\AA)$

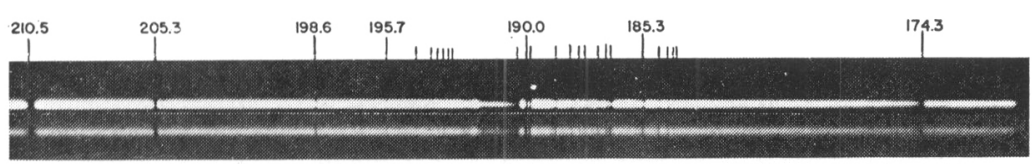

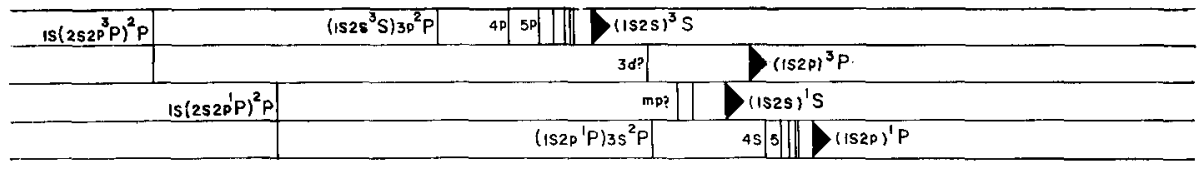

Fig. 6. Li I absorption spectrum on synchrotron-light background (after Ederer et al, 1970). 
Figure 6 shows the interesting $\mathrm{Li}$ I absorption spectrum arising from transitions of these types. The position of the deepest $1 s 2 s 2 p^{2} P$ level thus obtained confirms a recent calculation of A. Weiss (unpublished), to within $0.2 \mathrm{eV}$. The work on the $\mathrm{Li}$ I spectrum. has so far been done by use of a 'heat pipe', combined with thin-film Al windows to separate furnace from synchrotron and spectrographs. The experiments are continuing to embrace absorption cross-section measurements.

The $p$-shell absorption spectra of the remainder of the alkalis, ( $\mathrm{Na}$ I through $\mathrm{Cs}$ ), have already been explored by Connerade (Connerade et al., 1971; Connerade, 1970) and Mansfield (Mansfield and Garton, 1971), who variously employed the continuum of the 'BRV source' and the 'Hopfield' $\mathrm{He}_{2}$ continuum as background. Their work was considerably facilitated, in the windowless region between 300 and $700 \AA$, because of the excellent properties of a particular Bausch \& Lomb 3-metre grating. This grating provided with a platinum coating, was found usable to $300 \AA$ at normal incidence (Garton et al., 1969). Later experiments, with the same light source, at Harvard Observatory, have employed a grating from the same master, but gold-coated; in this case it was found possible to photograph the $\mathrm{He}_{\mathrm{I}}$ absorption resonance at $206 \AA$, previously reported by use of the synchrotron-light by Madden and Codling (1965).

In Figure 7 the $\mathrm{Na}$ I absorption spectrum (Connerade et al., 1971) is reproduced. Madden (unpublished) has re-examined this absorption spectrum, by use of the synchrotron-light, and has added a number of weak lines which it was not possible to identify with certainty from the earlier photographs with the 'BRV source'. Figure 8 shows the $\mathrm{K}_{\mathrm{I}}$ absorption spectrum (Mansfield and Garton, 1971). All these p-shell alkali absorption spectra show much confusion in structure, which is ascribable to

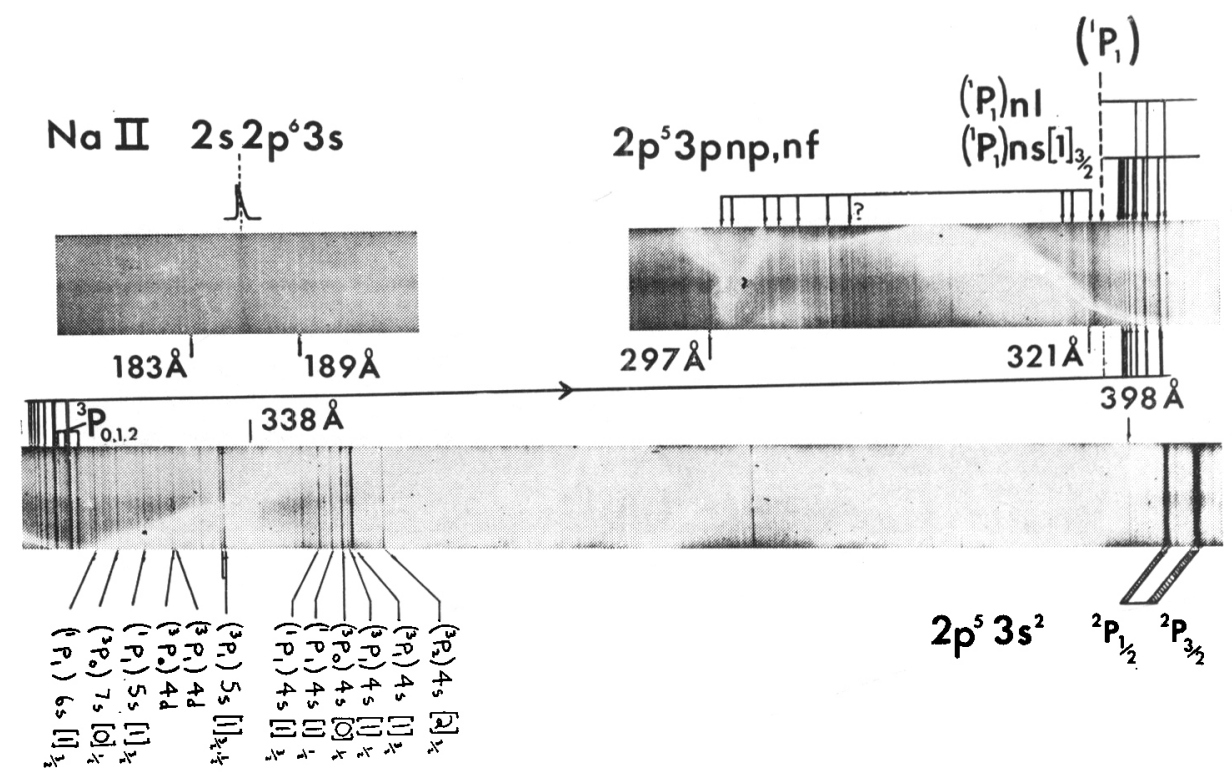

Fig. 7. NaI absorption spectrum on 'BRV-source' background (2-metre normal-incidence). 


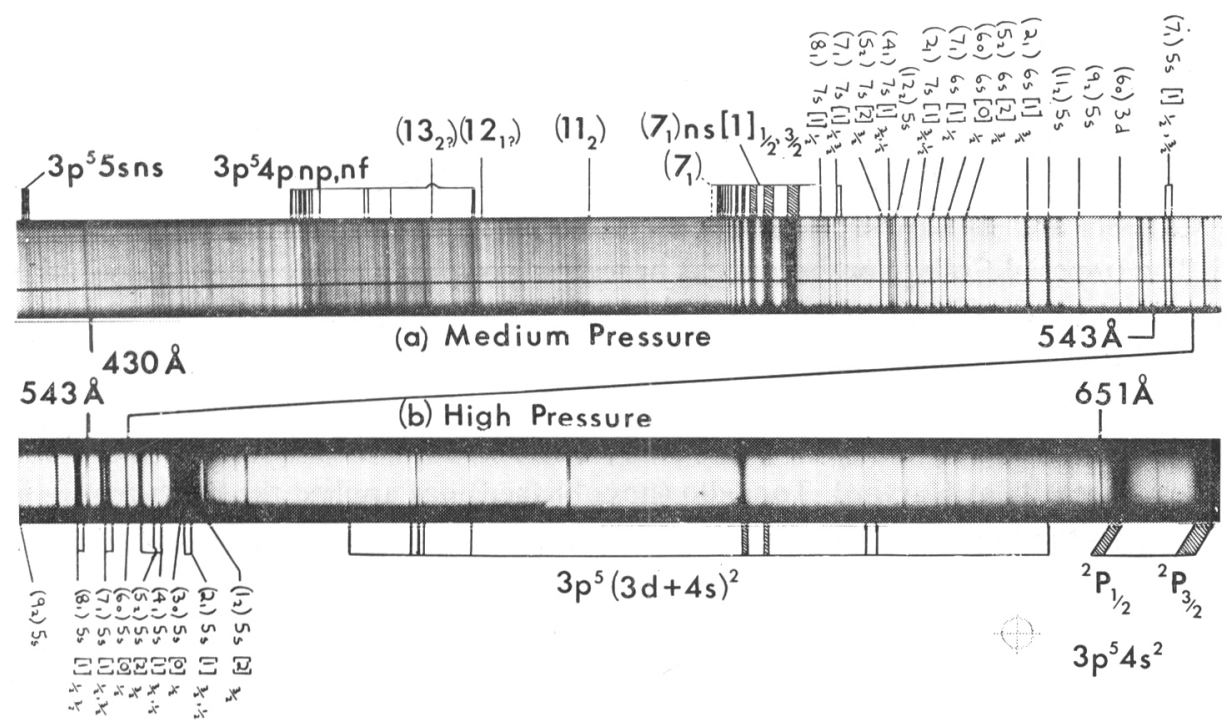

Fig. 8. K I absorption spectrum on 'BRV-source' background (3-metre normal-incidence).

configuration-mixing. In the case of the heavier alkalis ( $\mathrm{K}$ through $\mathrm{Cs}$ ) this mixing is traceable to severe overlapping of the low terms of the corresponding ions, eg: in RbI the $4 p^{5} 5 s$ and $4 p^{5} 4 d$ levels are badly intermingled. In Nal (Connerade et al., 1971) no such simple explanation can be found, and the configuration-mixing effects are more subtle. Moreover, the analyses of Rbi and Csi previously offered (Connerade, 1970), may need revision in view of very recent work on the spectra of the singly charged ions by Reader and Epstein (1970) at the Bureau of Standards.

The Imperial College programme of XUV absorption spectroscopy has also includ-

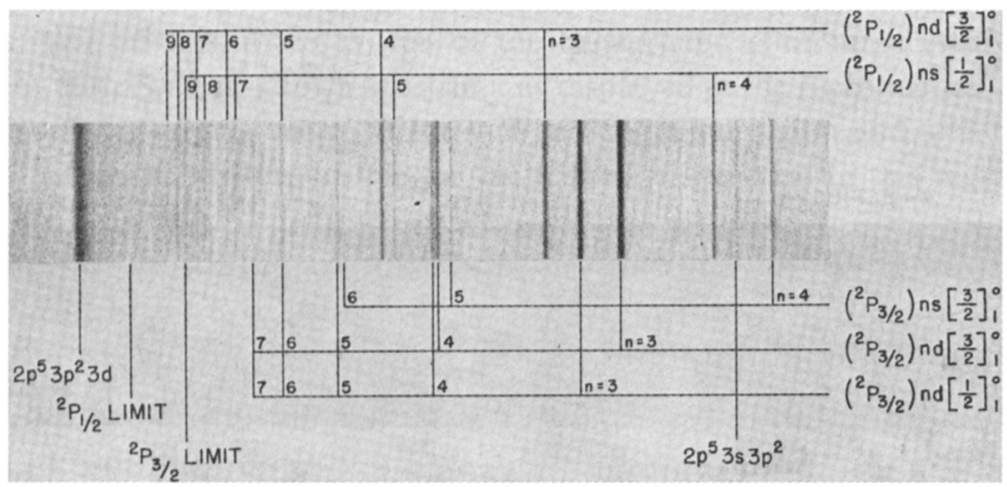

Fig. 9. MgI absorption spectrum on 'BRV-source' background (2-metre grazing-incidence). 
ed the alkaline-earth absorption spectra MgI through BaI. Figure 9 shows the first of these spectra obtained by Newsom (1971). In the case of MgI the greater part of the spectrum can be ascribed to transitions of the type $2 p^{6} 3 s^{2}{ }^{1} S_{0}--2 p^{5} 3 s^{2} n l$; however, the strongest feature in the whole spectrum lies at an energy higher than that of the $2 p^{5} 3 s^{2}{ }^{2} P$ limits, and must be ascribed to a multiple-electron jump. The spectra of Cal, SrI and BaI are in course of analysis by Dr Newsom and are as yet unpublished. The Imperial College programme is proceeding to photometric work on spectra of these kinds, by use of a specially built photoelectric split-beam spectrometer, designed for high speed scanning. The latter feature is a necessity because of the difficulty of maintaining a column of constant optical depth, of the metal vapour concerned, for a reasonable working time.

Very recently, at Harvard, Tondello (unpublished) has applied the 'flash-pyrolysis' method to the important case of FeI absorption, in the continuum beyond the first ionization potential at $1570 \AA$, and extending to about $800 \AA$. In addition to its astrophysical importance this element had the attraction that a theoretical prediction had been announced by Kelly and Ron (1971), concerning the positions of autoionization resonances and absorption cross-sections vs frequency. The first results of Tondello's work are shown in Figure 10 and, though it is too early to judge if there is much correspondence between the calculations and the observed absorption features, it is worth mention that the position of the broad and strong resonance at position $\mathrm{X}$

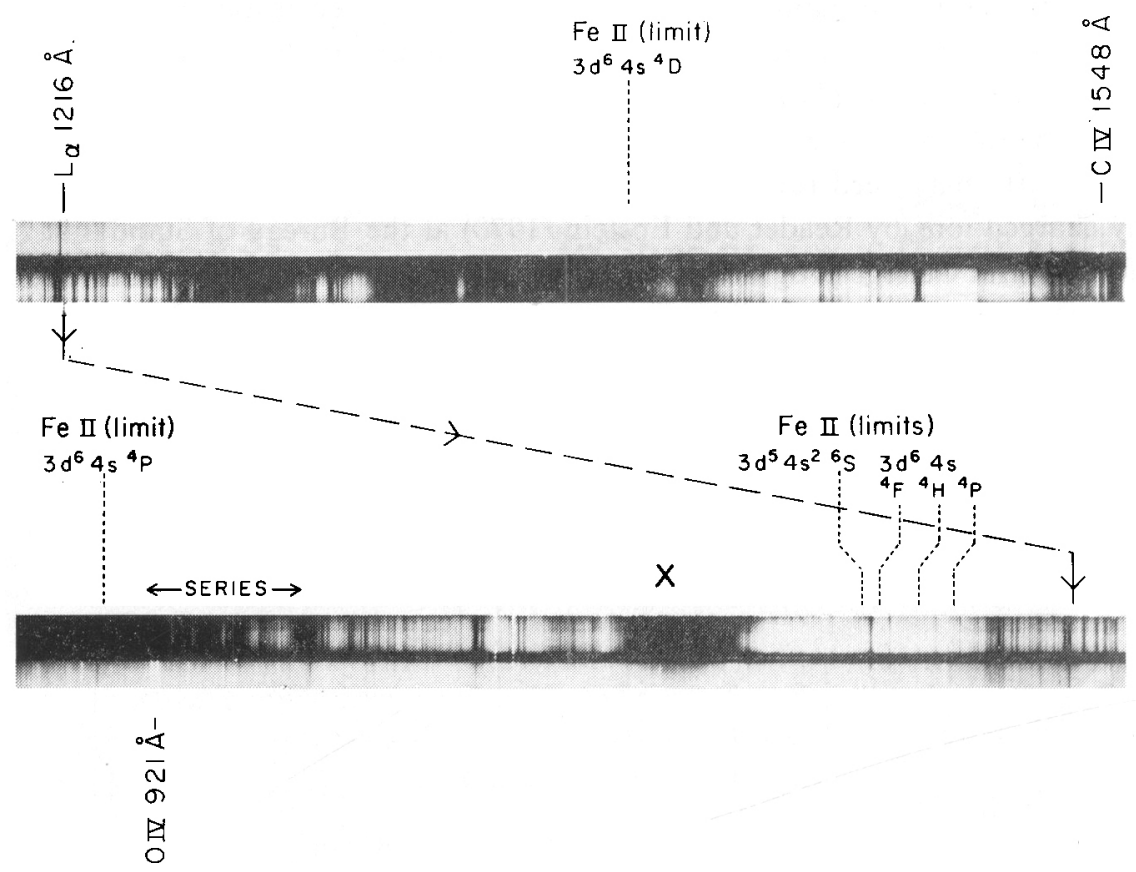

Fig. 10. Autoionization resonances in FeI. 
(Figure 10) agrees with the leading feature of the predicted spectrum of Kelly and Ron (1971). Further theory and experiment are doubtless needed in this case, and it is anticipated that the experimenters will soon be able to provide absolute absorption cross-sections.

Progress has also been made towards the observation of the inner-shell absorption spectra of ions. In some unpublished work, done several years ago at Harvard by Parkinson Reeves, Grasdalen and the author, a considerable extension of the LaII spectrum was photographed in the range above $1200 \AA$ and through the quartz ultraviolet, practically free from LaI absorption lines. The almost fully-ionized absorption path of lanthanum vapour was produced, in those experiments, by means of a pressure-driven shock-tube, and the limitation of spectral range was set by the LiF-optics employed. However, it was known that an interesting region, containing autoionization-resonance structure, would lie between 1000 and $1200 \AA$. Recently, again at Harvard, Dr Tondello has used the 'flash-pyrolysis' method to extend the La II absorption spectrum into this region. In point of fact, La II is technically the simplest case to be found in the Periodic Table, if we are searching for the absorption spectrum of an ion free from confusion from the neutral species. This spectrum also has the interest of arising from a structure isoelectronic with, but of very different properties from, the structure of BaI. Even more interest will attach to cases like singly-ionized alkali and alkaline-earth inner-shell spectra and the Harvard experiments are intended to include attempts to observe these. Similar objectives are being aimed for by the spectroscopists at CNRS in France, and Madame Mehlman-Balloffet describes elsewhere in these Proceedings successful experiments on ionic absorption spectra, which also depend upon use of the 'BRV source' to provide background continuum.

Some other highlights of recent work on atomic and molecular spectra in the far ultraviolet support the reflection that it is hardly possible to photograph a spectrum, even of a well-used light source like a $\mathrm{Hg}$ lamp or an iron arc, without finding some feature previously unnoticed or unexplained. An excellent example can be seen in a paper by Dalgarno et al. (1970), which concerns the well-known manyline spectrum of $\mathrm{H}_{2}$ emitted by a simple positive-column discharge. Practically every vacuum spectroscopist must have used such a light source at some time, either for purposes of adjustment or perhaps for provision of the background continuum emitted above $1650 \AA$. In this very well-known spectrum, as displayed in the reproduction of Figure 11 , some diffuse features occur, in the middle Schumann region and underlying the so-called Lyman bands responsible for the manyline spectrum in this region. These

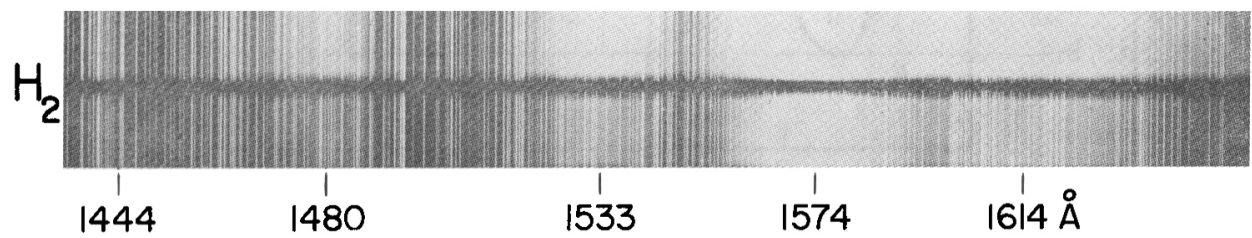

Fig. 11. $\mathrm{H}_{2}$ emission spectrum (after Dalgarno et al., 1970). 
diffuse features have been photographed frequently, - in the author's experience they must have occurred on several dozen plates, - but they remained ignored and uninterpreted. The interpretation of Dalgarno et al. (1970) is that they represent the first authenticated example of diffuse band structure occurring as a result of the overlap of the non-terminal part of the wave function of a lower potential-energy curve, with the wave function of the upper curve, as shown in Figure 12. The diffuse bands represent, in fact, the first recognized case of 'diffraction bands' predicted by Condon (1928) in his original discussion of the Franck-Condon Principle.

The programme of elegant experimental work, at fairly high resolution, at the Air Force Cambridge Research Laboratories continues, with reports of new results and interpretations. Thus, absorption spectra of $\mathrm{Ar}_{2}$ (Tanaka and Yoshino, 1970), obtained on a $6.65 \mathrm{~m}$ normal-incidence grating, have revealed nine band systems; the analysis gives a dissociation energy of the ground state of $76.9 \mathrm{~cm}^{-1}$. The intriguing absorption

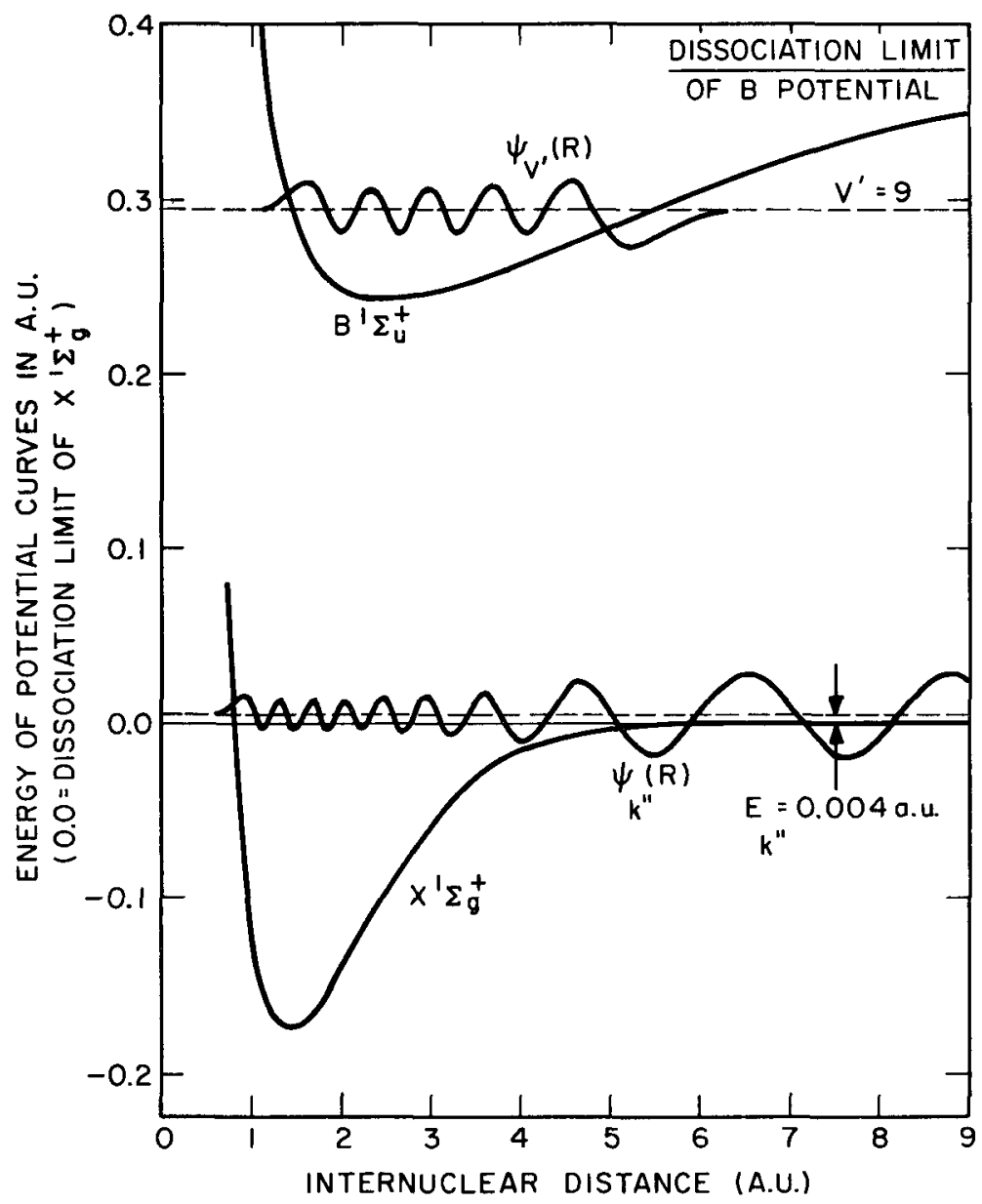

Fig. 12. Electronic states and wave-function character giving diffuse features of Figure 11. 
and emission features of $\mathrm{He}_{2}$, which lie in the range $500-1000 \AA$, have been further studied by the same authors (Tanaka and Yoshino, 1969).

Dr Y. Tanaka of AFCRL, has given the writer premission to mention some recent very interesting observations of the emission spectra of mixtures of the inert gases, as yet unpublished. To take a particular case, the spectrum of a positive-column discharge in a mixture of $\mathrm{Ne}$ with a few per cent of $\mathrm{Kr}$ (ie: the heavier inert gas in low concentration), shows four groups of fairly localized bands, each of which generally shows some structure under higher dispersion. The typical appearance of the bands is shown in Figure 13; as indicated, intervals occur between the different band groups which seem of the order of magnitude of the ${ }^{2} P$-splitting of both the NeII and KrII ions. Moreover, the spectral region in which the band group lies amounts to about the

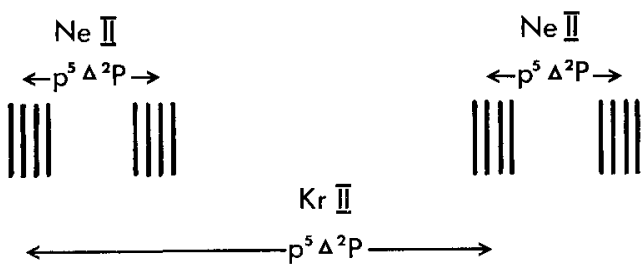

Fig. 13. Schematic diagram of emission hand groups observed in inert-gas mixtures (after Tanaka, unpublished).
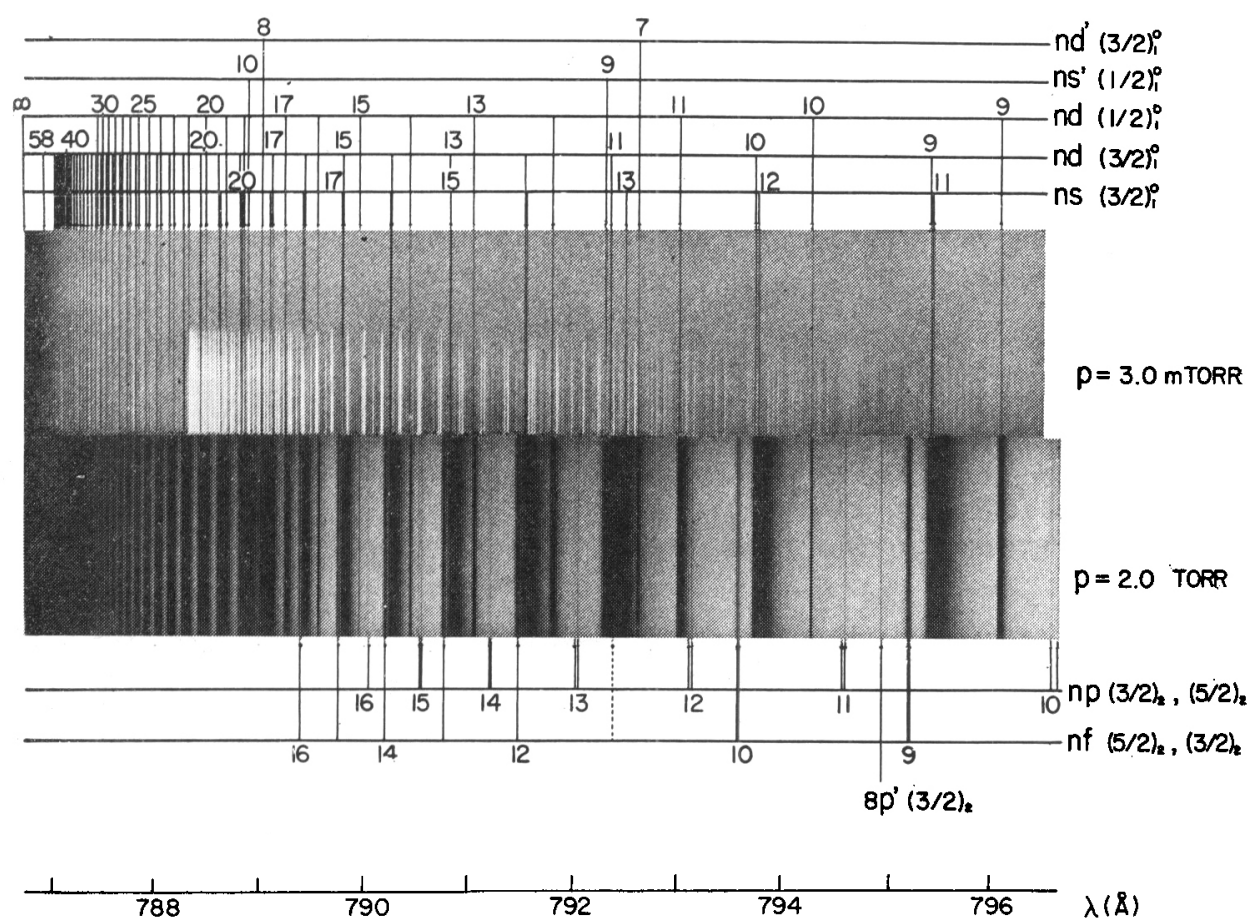

Fig. 14. Absorption series of Ar I (6.65 metre normal-incidence; after Yoshino, 1970). 
difference in ionization potential of neon and krypton. From these observations Tanaka tentatively ascribes the bands to radiative charge-exchange transitions in the molecular ion.

Some atomic absorption spectra have also been observed, at good resolution by the AFCRL group. A particularly impressive result is shown in Figure 14 (Yoshino, 1970), in the absorption spectrum of atomic argon. In addition to the five expected series, of character $3 p^{6}{ }^{1} S_{0}-3 p^{5} n p$ and $n f$, which extend to $n \sim 50$, the spectra have revealed six weak series which are identified as electric quadrupole transitions.

Another recent activity of novel character in molecular spectroscopy in the ultraviolet, concerns measurement of the distribution of oscillator strength over the rotational structure of electronic bands, by means of the 'hook method'. Through this valuable method of $f$-value determination has been considerably applied to atomic spectra (e.g. Penkin, 1964), the first application to molecular spectra was due to Anketell and Pery-Thorne (1967), who worked on the OH 0-0 band at $3060 \AA$. Pery-Thorne and Banfield have recently extended the technique to the important case of the NO $\gamma$-system which lies at shorter wavelenghts in the ultra-violet. The Imperial College programme under Pery-Thorne continues, with the expectation of $f$-value determinations for a number of metallic hydride molecules of astrophysical importance and the same technique is being applied at York University, Toronto (Professor R.W. Nicholls), to other molecules of astrophysical interest.

Some recent investigations at Harvard (Parkinson and Reeves, unpublished) again illustrate the remark that familiar spectra often contain interesting unexplained detail. The CdI arc spectrum is well known and, for instance, a reproduction of this at the end of Fowler's 'Report on Series in Line Spectra' (1922) shows the presence of a peculiar intensity discontinuity in the neighbourhood of $3100 \AA$, apparently superposed on a
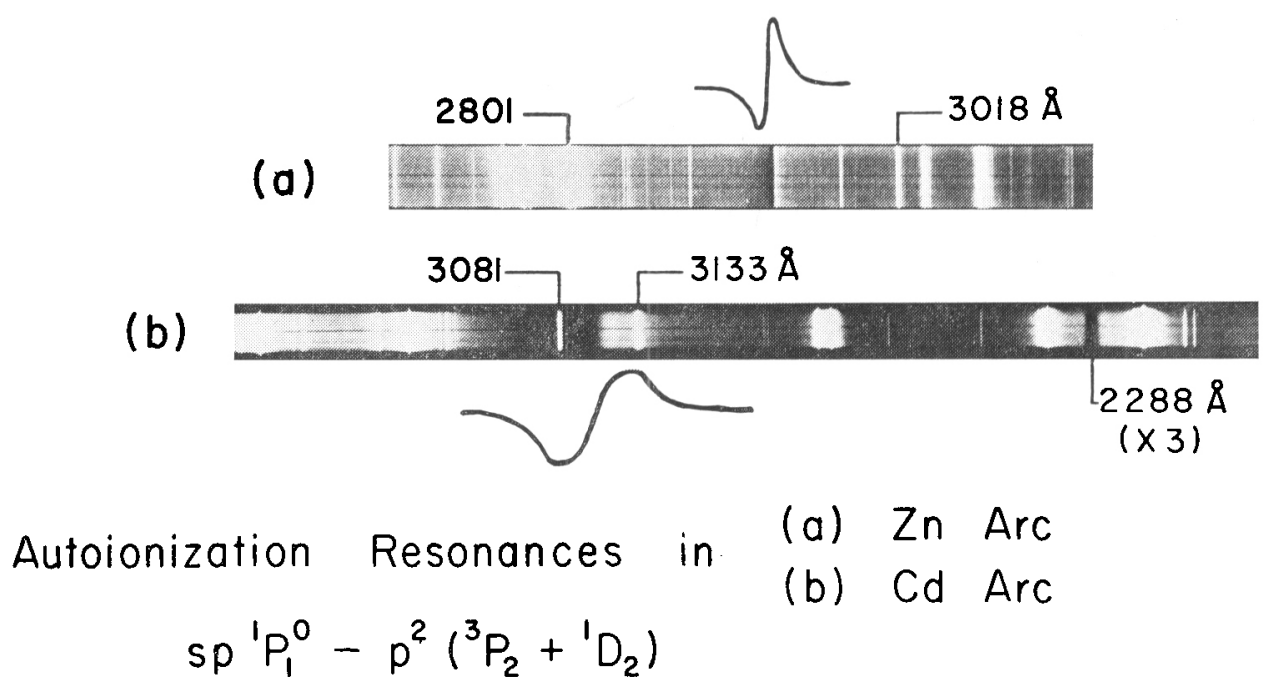

Fig. 15. 'Beutler-Fano resonances' in emission from metallic arcs in argon. 
recombination continuum. In the recent Harvard experiments, clearer pictures of this part of the Cdi spectrum were obtained when air was excluded from the arc, by surrounding it with a mantle of argon; this precaution eliminated a good deal of undesirable molecular structure from the spectrum. The result is seen in Figure 15b, the intensity peculiarity referred to being now much more clearly distinguishable. The feature is, in fact, an interesting example of a 'Beutler-Fano' resonance (Fano, 1961; Fano and Cooper, 1965), ascribable in this case to the transition from $5 s 5 p{ }^{1} P_{1}^{0}$ to the mixed state $5 p^{2}\left({ }^{3} P_{2}+{ }^{1} D_{2}\right)$; the position of the autoionizing upper level of this transition was already known from earlier work of Garton and Rajaratman (1955). Figure 15a represents the parallel case in $\mathrm{Zn}$, the resonance in question being of much smaller width.

\section{Solid State Vacuum Ultraviolet Spectroscopy}

Inclusion of material under this heading at a conference devoted to the spectroscopy of 'plasmas' can be justified (a) because measurements on absorption and reflection spectra combine with electron 'eigenlos' experiments in providing information on the occurrence of plasma oscillations in solids and (b) because of the rapid emergence of work in an area which may be described as 'solid state astrophysics', in relation to interstellar material.

Work on the far ultraviolet absorption and reflection spectra of solids is a burgeoning field, and such work combines with experimental studies of photoelectric emission, - including energy and angular analysis of the ejected electrons, two-photon absorption experiments etc. The group of workers at Hamburg and in Japan, in both cases by utilization of the polarized radiation from an electron synchrotron, have been especially active, and a number of other workers, - including the group at CNRS Laboratories, - have been applying ingenious polarizing devices in the study of the optical properties of solids. We now recognize that work in this field had, in order to be of much value, to await the emergence of ultra-high vacuum technology.

A comprehensive collaborative review of work in the field is in course of publication, as a book, by the group at the Bellevue Laboratories of CNRS in France (Vodar and Romand, 1971). The paragraphs following only focus on some work on the ultraviolet spectra of solids which connects with astrophysics rather directly, namely the absorption spectroscopy, and to less extent reflection spectroscopy, of 'matrix-isolated' atoms or molecules, ie: atoms or molecules laid down at low concentration in a selected solid matrix of relatively simple character such as a solid inert gas or cubic crystal of polar type or, - perhaps less simple, - a glassy material.

Experimental work in this field found its original stimulus from solid state physics, in particular from crystal-field theory. By the late 1960's the laboratory work became of astropyhsical importance, because of the possibility of explanation of the origin of the diffuse interstellar absorption features and the nature of the 'contamination' surrounding supernovae. A prominent experimental school, concerned in work of this kind, is at the Centre for Research in Experimental Space Science (York University, Toronto), where the programme of W.W. Duley includes the study of the optical absorp- 
tion spectra of metal-doped hydrocarbon and other matrices, and the absorption and reflection spectroscopy of simpler solids, like solid $\mathrm{N}_{2}, \mathrm{O}_{2}, \mathrm{CO}_{2}$, of possibly significant astrophysical abundance. Such work certainly has direct bearing on the extraordinary amount of new speculation recently appearing, as to the origin of the well-known interstellar absorption feature at $4430 \AA$ and its twenty or so companions. Recent theoretical and laboratory work, aimed at discovering the origin of these features, has produced some degree of simulation from all manner of solids, ranging from ice-coated graphite grains containing trapped cosmically abundant heavier atoms (Wickramsinghe et al, 1968; Hoyle and Wickramsinghe, 1967), $\mathrm{Ca}$ or Na atoms trapped in an organic matrix like $\mathrm{C}_{6} \mathrm{H}_{6}$ (Duley, 1969; Duley and Graham, 1969), or from dusts of complexes like garnets (Manning, 1970; Huffman, 1970). With such diversity of possibilities open, the spectra of the interstellar absorption features will be needed over a range of wavelengths from the infrared to the vacuum ultraviolet, and corresponding work must be continued in the laboratory. Figure 16 illustrates some possibilities of identification

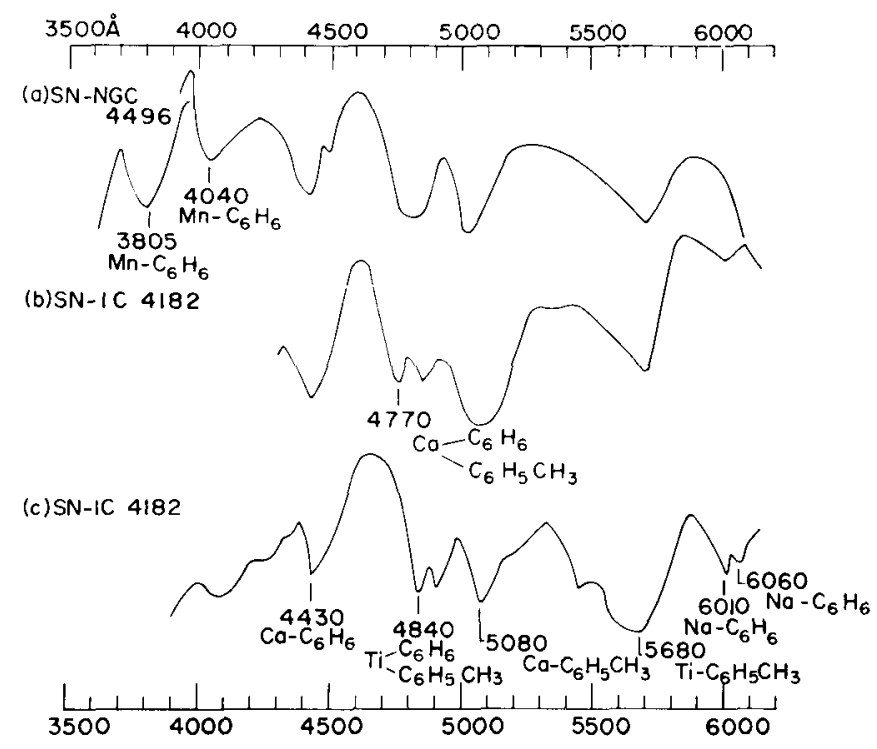

Fig. 16. Spectra of several supernovae with proposed identifications of diffuse absorption features (after Graham and Duley, 1971).

which have been suggested by Graham and Duley (1971) concerning the origin of diffuse absorption features associated with supernovae.

A very extensive and vigorous programme of work on vacuum ultraviolet solid state spectroscopy has been in progress on the Hamburg synchrotron, and many recent papers can be found referenced under R. Haensel and collaborators. Space permits mention here of but one of these publications under the title 'Line Shapes in Soft X-Ray Absorption Spectra of Solidified Rare Gases' (Haensel et al., 1970). In that paper the 
authors report observation of transitions in the solid from the $2 s$ sub-shell in $\mathrm{Ne}$ and the $3 s$ sub-shell in Ar, showing asymmetrical and 'window'-type Beutler-Fano profiles (Fano, 1961), similar to those observed in the corresponding gases (Madden and Codling, 1966), - the resonance line-shapes being preserved in the solid, with structures shifted to higher energies by the crystal fields.

\section{Spectroscopic Observations of Atomic Diamagnetism}

As in the case of the work briefly sketched in the foregoing section, the topic for discussion under this heading relates to experimental work which, though at the outset of no obvious astrophysical relevance, seems in course of acquiring such.

Many years ago van Vleck (1932) recognized that the effect of universal atomic diamagnetism would be to produce a shift of the whole of a Zeeman multiplet towards higher energies, by an amount proportional to the square of the magnetic field strength and, more significantly, proportional to the fourth power of the principal quantum number of the upper term. This effect was suitably named the 'Quadratic Zeeman Effect'. The first experiments demonstrating the occurrence of quadratic shifts were performed by Jenkins and Segré (1939), who observed the absorption spectrum of an alkali metal vapour, up to about the $n=35$ member, by use of a long absorption column placed within the poles of a cyclotron magnet. Their paper was accompanied by a theoretical explanation by Schiff and Snyder (1939), but the only other work, until very recently, was an examination of other alkali spectra by Harting and Klinkenberg (1949), again confirming the reality of the quadratic shifts.

About three years ago F. S. Tomkins and the writer, using a spectrograph of much higher resolution than was available to the earlier workers with the alkalis, (namely the Argonne $30 \mathrm{ft}$ Paschen-circle; Tomkins and Fred, 1954), examined long absorption series of Ba I (cf. Garton and Tomkins, 1969a), at a magnetic field of about $24 \mathrm{kG}$. Because of the better resolution available, and the fact that the oscillator strength in the Principal series of $\mathrm{Ba}$ i is much more smoothly distributed than in the corresponding series of an alkali, it was possible to observe the series to very high $n$ values, and much more detail of Zeeman patterns was discernible. The first results (Garton and Tomkins, 1969b) demonstrated the complexity of the effects of ' $l$-mixing', and ' $n$-mixing', in high series members and also led to observation of a quite new phenomenon, namely the existence in $\sigma$ polarization of apparently equally spaced resonances extending well into the region of the zero-field ionization continuum. Plainly, in the region of high $n$ values the motion of the radiating electron will be dominated by the magnetic field, the central field of the atomic core acting as a perturbation; this is the reverse of the ordinary Zeeman effect conditions. However, a satisfactory theoretical model has yet to be developed. Meanwhile, since the original absorption spectra in $\sigma$ polarization suffered from the disadvantage that both $\sigma$-components were present simultaneously, it was decided to attempt absorption spectra with the Barium column observed along the magnetic field, instead of transversely. To this end a large superconducting magnet, providing a working space at room temperature $2 \mathrm{ft}$ in length and $2 \mathrm{in}$. in diameter, 


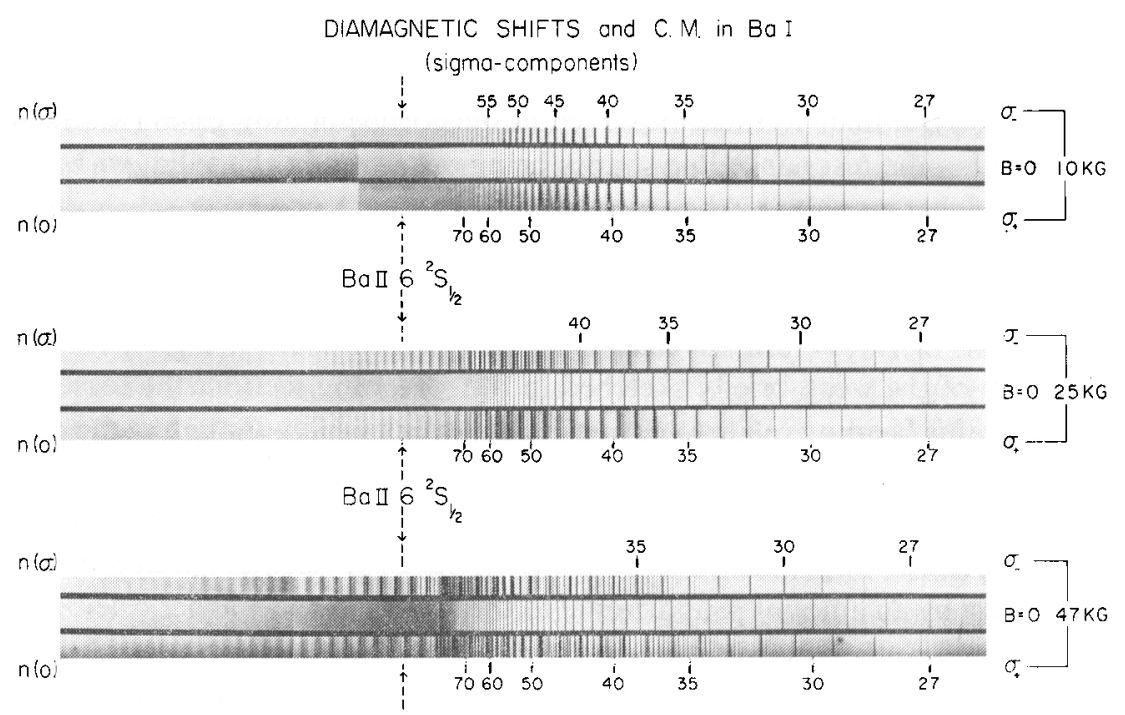

Fig. 17. Effects of diamagnetism in Bat absorption spectrum at several field strengths.

was constructed and the absorption spectrum of Bal re-observed. In this case it was possible to use a Babinet-compensator to seperate the two (circularly polarized) components of each $\sigma$ pair. The work was, moreover, carried out at a range of fields from 10 to $47 \mathrm{kG}$. Some of the first results are shown in Figure 17, from which can be seen plainly the occurrence of satellites due to $l$-mixing, the confusion due to ' $n$-mixing' and extensive structure well out into the zero-field ionization continuum. This work continues, with extension to other elements, and including photometric study of the 'continuum resonances'. The laboratory work appears of almost certain relevance to astrophysics, in view of the increasing number of papers (Kemp et al., 1970) Landstreet and Angel, 1971), on the possible existence of intense magnetic fields associated with some astronomical objects such as White Dwarfs.

\section{References}

Anketell, J. and Pery-Thorne, A.: 1967, Proc. Roy. Soc. A301, 343.

Balloffet, G., Romand, J. and Vodar, B.: 1961, Compt. Rend. Acad. Sci. Paris 252, 4139.

Bashkin, S.: 1970, Nucl. Instr. Methods 90, 3.

Beesley, M.J. and Casteldine, J.G.: 1970, Appl. Opt.9, 2720.

Bickel, W.S., Bergstrom, I., Buchta, R., Lundin, L. and Martinson, I.: 1969, Phys. Rev. 178, 118.

Bickel, W.S., Bashkin, S., Connerade, J.P. and Oona, H.: 1971, Opt. Soc. Amer., Spring Mecting

Programme, p. 11.

Bromander, J., Berry, H. G. and Curtis, L.T.: 1971, Opt. Soc. Amer. Spring Meeting Programme, p.12. Buchet, J.P., Denis, A., Desesquelles, J. and Dufay, M.: 1969, Phys. Letters 28A, 529.

Burch, J.M. and Palmer, D.A.: 1961, Optica Acta 8, 73.

Condon, E.U.: 1928, Phys. Rev. 32, 858.

Connerade, J.P.: 1970, Astrophys. J. 159, 685, 695. 
Connerade, J.P., Garton, W.R.S. and Mansfield, M.W.D. : 1971, Astrophys. J. 165, 203.

Cordelle, J., Flamand, J., Pieuchard, G. and Labeyrie, A.: 1969, in J.H. Dickson (ed)., Optical Instruments and Techniques, Oriel Press, Newcastle-upon-Tyne, p. 117.

Dalgarno, A., Herzberg, G. and Stephens, T.L.: 1970, Astrophys. J. 162, L49.

Damany, H., Roncin, I-Y. and Damany-Astoin: 1966, Appl. Opt. 5, 297.

Drake, G.W.F. and Dalgarno, A.: 1970, Phys. Rev. A1, 1325.

Dufay, M.: 1970, Nucl. Instr. Methods 90, 15.

Duley, W.W.: 1969, Physica 41, 134.

Duley, W.W. and Graham, W.R.M.: 1969, Nature 224, 785.

Eastman, Y.: 1968, Sci. Amer. 218, 38.

Ederer, D.L., Lucatorto, T. and Madden, R.P.: 1970, Phys. Rev, Letters 25, 1537.

Fano, U.: 1961, Phys. Rev. 124, 1866.

Fano, U. and Cooper, J.W.: 1965, Phys. Rev. 137, A1364.

Fowler, A.: 1922, Report on Series in Line Spectra, Physical Society of London, Fleetway Press.

Franks, A.: 1970, Applied X-Rays, Electrons and Ions, New Delhi Press.

Gahwiller, C., Brown, F.C. and Fujita, H.: 1970, Rev. Sci. Instr. 41, 1275.

Garcia, J.D.: 1971, Opt. Soc. Amer. Spring Meeting Programme, p. 11.

Garton, W.R.S. and Rajaratman, A.: 1955, Proc. Phys. Soc. A68, 1107.

Garton, W.R.S. and Tomkins, F.S.: 1969a, Astrophys. J. 158, 1219.

Garton, W.R.S. and Tomkins, F.S.: 1969b, Astrophys. J. 158, 839.

Garton, W.R.S., Connerade, J.P., Mansfield, M.W.D. and Wheaton, J.E.G.: 1969, Appl. Opt. 8, 919. Graham, W.R.M. and Duley, W.W.: 1971, Nature 232, 43.

Grover, G.M., Cotter, T.P., and Erickson, G.F.: 1964, J. Appl. Phys. 35, 1990.

Haensel, R., Keitel, G., Kunz, C. and Schreiber, P.: 1970, Phys. Rev. Letters 25, 208.

Harrison, G.R. and Thompson, S.W.: 1970, J. Opt. Soc. Amer. 60, 591.

Harting, D. and Klinkenberg, P.F.A.: : 1949, Physica 14, 669.

Hoyle, F. and Wickramsinghe, N.C.: 1967, Nature 214, 969.

Huffman, D.R.: 1970, Astrophys. J. 161, 1157.

Jenkins, F.A. and Segré, G.: 1939, Phys. Rev. 55, 52.

Kelly, H. and Ron, A.: 1971, Phys. Rev. Letters 26, 1359.

Kemp, J.C., Swedlund, J.B., Landstreet, J.D. and Angel, J.R.P.: 1970, Astrophys. J. Letters 161, L77.

Keubler, N.A. and Nelson, L.S.: 1961, J. Opt. Soc. Amer. 51, 1411.

Labeyrie, A. and Flamand, J.: 1969, Opt. Comm. 1, 1.

Landstreet, J.D. and Angel, J.R.P.: 1971, Astrophys. J. Letters 165, L67.

Liu, C.H., Bashkin, S., Bickel, W.S. and Hadeishi, T.: 1971, Opt. Soc. Amer., Spring Meeting Programme, p. 12.

Lotte, B., Bon, M. and Romand, J.: 1963, J. Phys. 24, 346.

Madden, R.P. and Codling, K.: 1965, Astrophys. J. 141, 364.

Madden, R.P. and Codling, K.: 1966, in A. Temkin (ed.), Autoionization, Mono Book Corp. Baltimore.

Manning, P.G.: 1970, Nature 227, 1121.

Mansfield, M.W.D. and Garton, W.R.S.: 1971, in press.

Marrone, P.V. and Wurster, W.H.: 1971, J. Quant. Spectr. Radiative Transfer 11, 327.

Martinson, I.: 1971, Opt. Soc. Amer., Spring Meeting Programme, p. 12.

Mather, J.W.: 1964, Phys. Fluids 8, 366.

Nelson, L.S. and Kuebler, N.A.: 1962, J. Chem. Phys. 37, 47.

Newsom, G.H.: 1971, Astrophys. J. 166, 243.

Penkin, N.P.: 1964, J. Quant. Spectr. Radiative Transfer 4, 41.

Pery-Thorne, A. and Banfield, F.: 1970, J. Phys. B3, 1011.

Reader, J. and Epstein, G.L.: 1970, J. Opt. Soc. Amer. 60, 713, and private communication.

Schiff, L.I. and Snyder, H.: 1939, Phys. Rev. 55, 59.

Speer, R.J.: 1970, in B. I. Henke, J.B. Newkirk and G.R. Mallett (eds.), Adv. X-Ray Analysis 13, 382, Plenum Press, New York.

Speer, R.J.: 1971, Proc. XIIth Scottish Universities Summer School, Wiley, London, in press.

Tanaka, Y. and Yoshino, K.: 1969, J. Chem. Phys. 50, 3087.

Tanaka, Y. and Yoshino, K.: 1970, J. Chem. Phys. 53, 2012.

Tomkins, F.S. and Fred, M.: 1954, Spectrochem. Acta 6, 139.

van Vleck, J.H.: 1932, The Theory of Electric and Magnetic Susceptibilities, Oxford Univ. Press. 
Vodar, B. and Romand, J.: 1971, Some Aspects of Vacuum Ultraviolet Radiation Physics, Pergamon Press, forthcoming publication.

Wickramsinghe, N.C., Ireland, J.G., Nandy, K., Seddon, H. and Wolstencroft, R.D.: 1968, Nature 217, 412 .

Yoshino, K.: 1970, J. Opt. Soc. Amer. 60, 1220.

\section{DISCUSSION}

H.G. Van Bueren: Is $50 \mathrm{kG}$ a strong enough field to explain the periodic structure above the ionization limit by assuming the electrons to be essentially experiencing only the magnetic field?

W.R.S. Garton: Oh yes! Remember that we are here concerned with electrons in very high 'orbits'; they will have motions controlled much more by $\mathbf{B}$ than by the central field of the atomic core.

A.H. Gabriel: I was interested to see the spectra you showed of the $1 s 2 s n p$ series of LiI, obtained by Dr R. Madden's group. These are isoelectronic with those responsible for the soft X-ray satellite emission lines. Is he able to measure the widths? This information would supplement the rather incomplete theory available for the autoionization rates.

W.R.S. Garton: He is now measuring these, but at the short wavelengths involved, the experimental precision may limit the accuracy of the values obtained for some of the transitions. However, the stronger and broader resonances will soon be measured photometrically.

$V$. Kotov: Is the sense of $\sigma$ spectrum shift dependent on the direction of the magnetic field? What is the ratio of this shift to the field strength? In the sunspots we have also some shift of the circularly polarized spectra changing its sense with change of the field direction. Of course in the sunspots the field is very small compared with that discussed here. What reasons can there be for this shift?

W.R.S. Garton: The quadratic shifts which account for the situation for the lower values of $n$ are towards higher energy - shorter wavelength, - independent of direction of B. It seems unlikely that quadratic shifts will find relevance to solar or sunspot spectra. At fields of a few $\times 10^{3}$ Oe much higher values of $n$ than $\sim 16$ observed in sunspots are needed. The only references to quadratic effect in astrophysics, to my knowledge, are in recent papers by Preston and Thrimble, concerned with White Dwarf Spectra. 